\title{
Monitoring of soil moisture and irrigation forecast under the condition of drip irrigation in greenhouse
}

\author{
Yuan Wang ${ }^{1}$, Yangren Wang ${ }^{1 *}$, Hao Wang ${ }^{1}$ and Chengqi Gong ${ }^{1}$ \\ ${ }^{1}$ Department of Water Conservancy Engineering, Tianjin Agricultural University, Tianjin 300384, China
}

\begin{abstract}
The accurate prediction of the soil moisture in greenhouse can improve the accuracy of irrigation forecast, thus saving water and increasing production. In this paper, the monitoring data were obtained from the greenhouse in Agricultural Demonstration Park of Northland Spring, Wuqing District, Tianjin. The soil moisture was predicted based on the GM $(1,1)$ model, and the effect of water-saving and increased production was tested with the results of the economic irrigation research in the same area. The results show that the GM $(1,1)$ model has a higher accuracy of soil moisture prediction. The correlation coefficient $\left(\mathrm{R}^{2}\right)$ of the predicted value and the measured value is higher than 0.9 ; the small error probability $\mathrm{P}$ is 1 ; the mean relative prediction errors are all less than 0.1 . Therefore, the GM $(1,1)$ model can be used to predict soil moisture content.
\end{abstract}

\section{Introduction}

Soil moisture refers to water content of the root layering of the crop ${ }^{[1]}$. Collecting data related to soil moisture and weather and other factors by monitoring instruments, the moisture prediction in the next period of time can be achieved by mathematical modeling in soil moisture monitor and forecast. The irrigation forecast is a prediction based on the crop water requirement, soil moisture status, weather forecast and other conditions for the date of irrigation. Soil moisture monitor and irrigation forecasting technology can save water and increase production, ensuring the normal growth and development of crops, and maximizing economic benefits. It is a relatively low-cost technology to increase production and income. Domestic scholars have had such research on soil moisture and irrigation forecast. The research results of Sun Kai et al. showed that the change of soil moisture is related to location ${ }^{[2]}$. Research on soil moisture monitoring, soil moisture prediction, and irrigation forecasting has been carried out for eggplant, tomato and cucumber under drip irrigation conditions in the greenhouse of Agricultural Demonstration Park of Northland Spring, Wuqing District, Tianjin.

\section{Materials and Method}

\subsection{Soil moisture monitoring}

\subsubsection{Moisture}

Soil moisture, that is, the content of soil water. Soil moisture is divided into surface $(0-20 \mathrm{~cm})$, bottom layer $(20-50 \mathrm{~cm})$, base layer $(50-100 \mathrm{~cm})$ and deep moisture $(100-200 \mathrm{~cm})$ according to the relationship between crop root development and water. The surface soil moisture has more chances to increase and decrease in the period of crop growth. The bottom layer moisture has the strongest root absorbed water most during the growth period. The basement soil moisture changes significantly with the seasons during the year, but the interannual variation is not obvious. It plays a significant role in crop growth and development under dry seasons when there is a shortage of water. The deep moisture change is not obvious.

\subsubsection{Data collection}

This study uses a wireless soil moisture monitoring system, including 3 nodes and a base station (for receiving soil moisture content sent by the node, sent to the management center via the Internet), and the base station controls three nodes (collecting soil moisture content data, transmitting to the base station via wireless.), Figure1(a). Each node is connected to two soil moisture sensors. The two sensors are buried at $20 \mathrm{~cm}$ and $40 \mathrm{~cm}$ below the surface, both located below the drip irrigation belt, as are shown in Figure1(b). Based on the Internet, the automatic collection and transmission of soil moisture data can be achieved, realizing 24-hour continuous online monitoring and transmitting the data to the management center (a desktop computer). The data is properly processed and reports are generated, the irrigation forecast information is transmitted to users' mobile phones via SMS.

\footnotetext{
*Corresponding author's e-mail: wyrf@163.com
} 


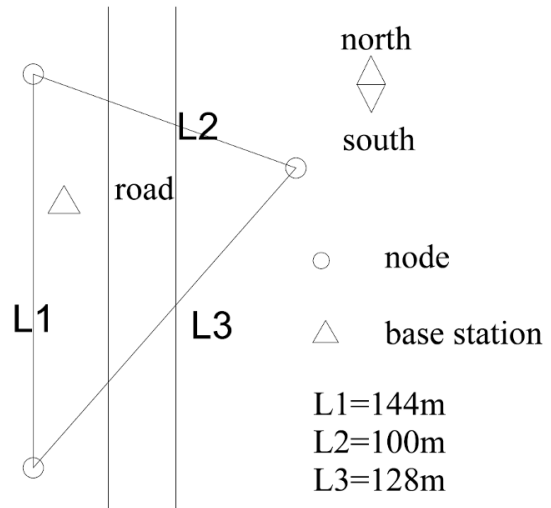

(a)

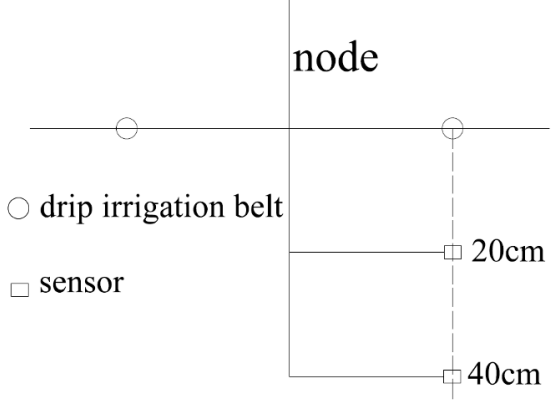

(b)

Figure 1. Schematic diagram of base station and node sensor arrangement

\subsection{GM $(1,1)$ method}

The mechanistic study showed that the changes of soil moisture content in greenhouses were affected by irrigation, deep soil moisture, groundwater, inter-plant evaporation, crop transpiration and deep seepage, etc. ${ }^{[3]} \mathrm{In}$ addition, the change of soil moisture content is also affected by some unknown factors whose influence is uncertain. The gray prediction is suitable for uncertain system prediction ${ }^{[4]}$. Therefore, this paper adopts the GM $(1,1)$ method to predict soil moisture in the short term.

The basic principle of gray prediction is to find the law of system change by using the original data, thus generating a data sequence with strong regularity, and then establishing a corresponding differential equation model to predict the future development trend of things.

$\mathrm{GM}(1,1)$ is a typical method in gray prediction theory. The modeling steps are as follows:

(1) Determine the level ratio of the original sequence $\mathrm{X}^{(0)}$, and the calculation formula is,

$$
\lambda(k)=\frac{x^{(0)}(k-1)}{x^{(0)}(k)}, k=2,3, \ldots, n
$$

If all the level ratio fall within the allowable range, $\lambda=\left(e^{\frac{-2}{n+1}}, e^{\frac{2}{n+1}}\right), \quad \mathrm{GM}(1,1)$ model can be established by using the sequence $\mathrm{X}^{(0)}$, Otherwise, it is necessary to do appropriate transformation processing on the data, such as translation transformation,

$$
y^{(0)}(k)=x^{(0)}(k)+c, k=1,2, \ldots, n
$$

The value of $\mathrm{c}$ is such that the level ratio of the data falls within the allowable range.

(2) Perform an accumulation process on the original sequence to obtain a new sequence

$$
X^{(1)}(k)=\sum_{n=1}^{k} X^{(0)}(n)
$$

(3) The trend of the new series $X^{(1)}$ can be approximated as a differential equation

$$
\frac{\mathrm{d} X^{(1)}}{\mathrm{d} t}+a X^{(1)}=u
$$

In the formula $a$ - develop gray number, u endogenous control gray number.

(4) Model solving

The predictive model can be expressed as

$$
\begin{aligned}
& Y_{n}=B \hat{\alpha} \\
& \mathbf{Y}_{n}=\left[\begin{array}{llll}
X^{(0)}(2) & X^{(0)}(3) & \cdots & X^{(0)}(n)
\end{array}\right]^{\mathrm{T}} \\
& \mathbf{B}=\left[\begin{array}{cc}
-\frac{1}{2}\left(X^{(1)}(1)+X^{(1)}(2)\right. & 1 \\
-\frac{1}{2}\left(X^{(1)}(2)+X^{(1)}(3)\right. & 1 \\
\vdots & \vdots \\
-\frac{1}{2}\left(X^{(1)}(n-1)+X^{(1)}(n)\right. & 1
\end{array}\right] \\
& \hat{\alpha}=\left[\begin{array}{l}
a \\
u
\end{array}\right]
\end{aligned}
$$

In the formula, $\hat{\alpha}$ is the parameters to be estimated, and $\widehat{\alpha}$ is solved by the least squares method.

$$
\hat{\alpha}=\left(\mathrm{B}^{\mathrm{T}} \mathrm{B}\right)^{-1} \mathrm{~B}^{\mathrm{T}} \mathrm{Y}_{n}
$$

Discrete time response function based on parameter variables

$$
\hat{X}^{(1)}(t+1)=\left(\hat{X}^{(0)}(1)-\frac{u}{a}\right) \mathrm{e}^{-\alpha t}+\frac{u}{a}
$$

$\hat{X}^{(1)}(t+1)$ is the accumulated predicted value which is recovered, as follows,

$$
\hat{X}^{(0)}(t+1)=\hat{X}^{(1)}(t+1)-\hat{X}^{(1)}(t)
$$

(5) Model test

The test method of the gray prediction model uses two ways: the residual test and the posterior difference test. The residual test process is to obtain the predicted value, the absolute error and the relative error respectively, and then calculate the Mean relative error (MRE) to judge the prediction accuracy of the model. If $|M R E|<0.1$, it is considered to achieve a higher accuracy requirement; Otherwise, if $|M R E|<0.2$, it is considered to achieve a general accuracy requirement. The posterior difference test method mainly calculates the small error probability $\mathrm{P}$, and then compares the test index $\mathrm{T}$ with the gray prediction accuracy level standard, as shown in Table 1. 
Table 1. Test index $T$ value and gray prediction accuracy test level standard

\begin{tabular}{ccccc}
\hline Test index & Level1(excellent) & Level 2 (good) & Level 3 (moderate) & Level 4 (poor) \\
\hline $\mathrm{T}$ & $>0.85$ & $0.75-0.85$ & $0.65-0.75$ & $0.50-0.65$ \\
\hline
\end{tabular}

\subsection{Determination of the economic irrigation lower limit and irrigation forecast}

The traditional lower limit of suitable soil water content refers to the minimum soil water content allowed in the planned wet layer that does not inhibit the normal growth of crops. According to the lower limit, the maximum yield of crops can be achieved, which is suitable for determining the irrigation time under sufficient water supply conditions ${ }^{[5]}$. The lower limit of economic irrigation refers to the adjustment of the economic irrigation schedule with the purpose of gaining maximum economic benefit per unit area. The soil moisture content and the corresponding time before the irrigation in the economic irrigation schedule are plotted as curves. The curve is the economic irrigation lower limit. The soil moisture content is

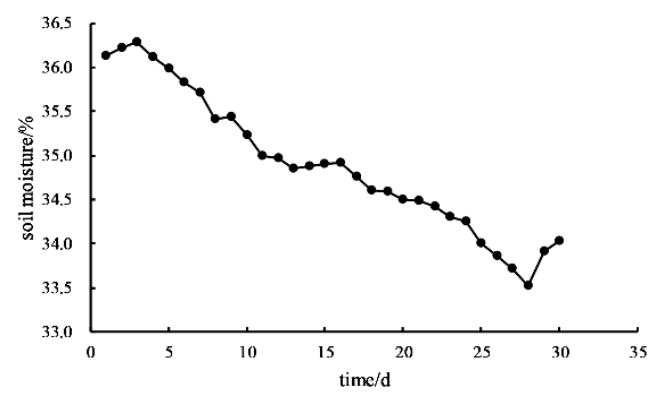

(a) $20 \mathrm{~cm}$ monitored by sensor and prediction irrigation. When the soil moisture content is predicted to reach the irrigation limit, the user is sent an warning message which is to remind the user to make irrigation schedule.

\section{Results and analysis}

\subsection{Analysis of soil moisture changes}

For the three crops: tomato, eggplant and cucumber, the moisture monitoring system was used to monitor the change from planting to pulling.

According to the soil moisture monitoring data of cucumber, tomato and eggplant in November 2017, the moisture trends of the three crops were plotted, as shown in Figure 2, Figure 3 and Figure 4, respectively.

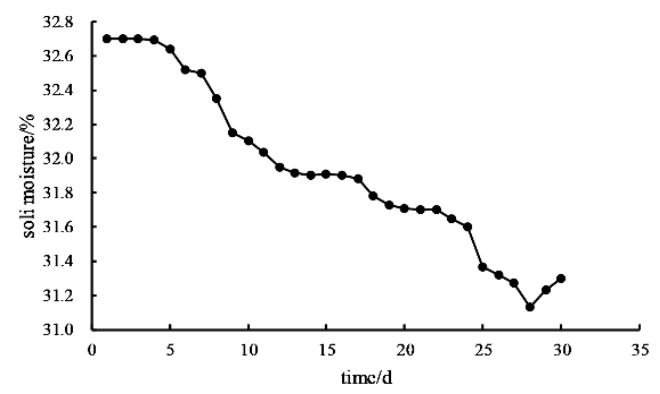

(b) $40 \mathrm{~cm}$

Figure 2. The moisture change of cucumber (2017.11)

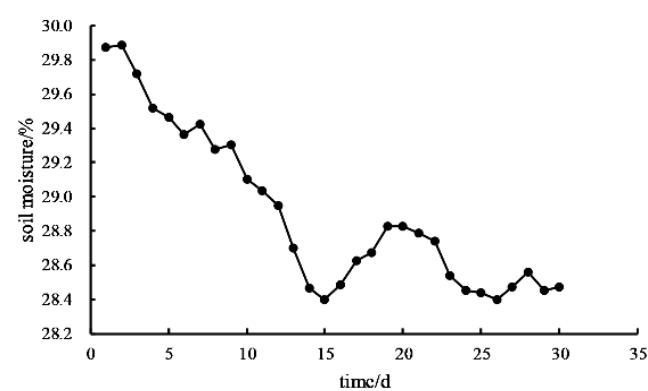

(a) $20 \mathrm{~cm}$

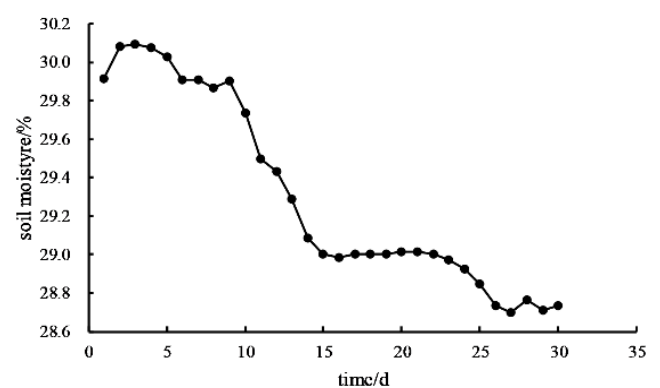

(b) $40 \mathrm{~cm}$

Figure 3. The moisture change of tomato (2017.11)

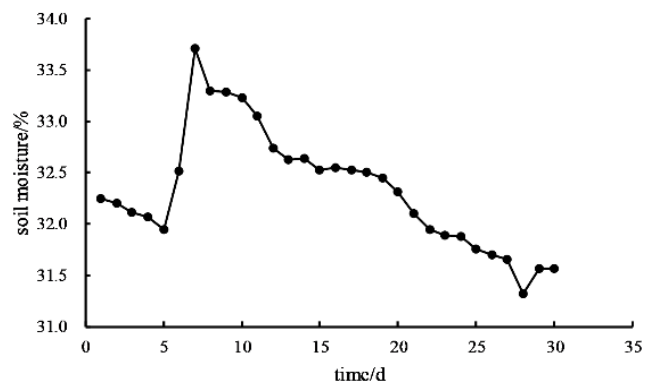

(a) $20 \mathrm{~cm}$

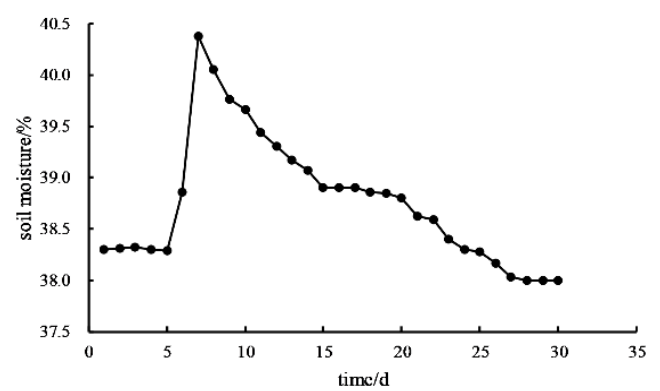

(b) $40 \mathrm{~cm}$

Figure 4. The moisture change of eggplant (2017.11) 
It can be seen from Figure 2 that the previous irrigation of cucumber made the soil moisture content reach the maximum on the third day of the month, and the soil moisture content reached the minimum on the 28th day. From the third day to the 28th day, the soil moisture content is basically decreasing day by day. It can be seen from Figure 3 that the previous watering of tomato made the soil moisture content reach the maximum on the second day of the month, and the soil moisture content reached the minimum on the 15 th day, and the irrigation was carried out again on 15th day. The soil moisture content decreased gradually from 2 nd day to 15 th day. It can be seen that the eggplant was irrigated on the fifth day from Figure4, and the soil moisture content decreased gradually from day 5 to day 27 .

\subsection{Analysis of prediction results}

The data obtained by the monitoring system are of the same order of magnitude and the amplitude of variation is similar, so that any monitoring data in a continuous period(during neighbor two irrigation) can be used as the original data sequence. This paper selects the soil moisture monitoring data in November 2017 as the original data sequence for the analysis. The soil moisture data that are not affected by irrigation should be used for gray prediction. For the data of November 2017, from the third day to the 28th day, it is for the cucumber; from the second day to the 15 th day, for the tomato; from the seventh day to the 27 th day, for the eggplant.

The original data sequence is processed by the MATLAB program for level comparison to obtain the level ratio $\lambda . \lambda$ is shown in Table 5. The required level ratios fall within the allowable range $\lambda=\left(e^{\frac{-2}{n+1}}, e^{\frac{2}{n+1}}\right)$, so the selected data sequence can be modeled by $\operatorname{GM}(1,1)$.

Table 5. Gray prediction model level ratio

\begin{tabular}{cccccccccc}
\hline \multirow{2}{*}{ Crops } & Location of & \multicolumn{2}{c}{ Calculated $\lambda$} & \multicolumn{2}{c}{ Allowable } & \multicolumn{2}{c}{ Parameters } & \multicolumn{2}{c}{ Evaluation indicators } \\
\cline { 3 - 9 } & sensor & Min & Max & Min & Max & a & u & MRE & P \\
\hline Cucumber & $20 \mathrm{~cm}$ & 0.9975 & 1.0082 & 0.90 & 1.11 & 0.0031 & 36.39 & $1.08 \times 10^{-5}$ \\
Cucumber & $40 \mathrm{~cm}$ & 0.9968 & 1.0073 & 0.90 & 1.11 & 0.0017 & 32.75 & $8.74 \times 10^{-6}$ & 1 \\
Tomato & $20 \mathrm{~cm}$ & 0.9980 & 1.0087 & 0.87 & 1.14 & 0.0035 & 29.90 & $1.27 \times 10^{-5}$ & 1 \\
Tomato & $40 \mathrm{~cm}$ & 0.9990 & 1.0078 & 0.87 & 1.14 & 0.0031 & 30.37 & $1.23 \times 10^{-5}$ & 1 \\
Eggplant & $20 \mathrm{~cm}$ & 0.9921 & 1.0123 & 0.92 & 1.08 & 0.0026 & 33.38 & $1.02 \times 10^{-5}$ & 1 \\
Eggplant & $40 \mathrm{~cm}$ & 1.0000 & 1.0082 & 0.92 & 1.08 & 0.0022 & 39.87 & $6.32 \times 10^{-5}$ & 1 \\
\hline
\end{tabular}

According to the GM $(1,1)$ modeling step, the MATLAB program is written to obtain the model parameters and predict. The model average relative error (MRE) and the small error probability $\mathrm{P}$ value are calculated to test the model accuracy. The results of the model parameters and evaluation indicators are shown in Table 5.

It can be seen from Table 5 that the $|M R E|$ of the selected 6 sets of data sequences are all less than 0.1 , and the prediction accuracy reaches a higher requirement; the $P$ values are all 1 and are higher than 0.85 , and the level of model accuracy is superior.

Each data sequence measured obtained by monitoring and the values predicted by the GM $(1,1)$ model are plotted as a scatter plot in Excel, and the trend line is added to display the correlation coefficient $\mathrm{R}^{2}$.

By comparing the correlation coefficient between measured and predicted moisture of cucumber, eggplant and tomato, we found that all $\mathrm{R}^{2}$ are higher than 0.9(in $20 \mathrm{~cm}, 40 \mathrm{~cm}$ for cucumber, eggplant and tomato, respectively, 0.9450, 0.9577, 0.9145, 0.9403, 0.9680, 0.9680 ), so the effect of prediction is good. Based on the judgment of all the evaluation indicators, $\operatorname{GM}(1,1)$ model can be used for soil moisture prediction and water irrigation forecast.

The obvious water-saving and income-increasing effects were obtained by the irrigation forecast using the GM $(1,1)$ model to predict soil moisture based on the conclusions of Wang Hao's research ${ }^{[6]}$ on the economic irrigation lower limit in the same area (Agricultural Demonstration Park of Northland Spring, Wuqing District, Tianjin).

\section{Conclusion}

The GM $(1,1)$ model has a good prediction result for soil moisture. The average relative error (MRE) is less than 0.1 , all of the test index $\mathrm{T}$ is 1 , the correlation coefficient $\mathrm{R}^{2}$ of the soil moisture content measured and the predicted is higher than 0.9 .

Compared with the existing actual irrigation schedule, the irrigation forecast based on the economic irrigation lower limit has obvious effect of saving water and increasing income.

\section{Acknowledgements}

Funds for this research was provided by the National Natural Science Foundation of China (51779174), and Students' Innovation and Entrepreneurship Training Program (201810061021).

\section{References}

1. Li, M.S. (2005) The study on prediction model of soil moisture[D] Hehai University, 11-27.

2. Zhang, Z.Y. (2014) Soil Moisture Forecast and Crops Irrigation Schedule Multi-Objective Optimization. [D] 
Agricultural University of Hebei.

3. Fan, X.R., Wang, Y.R., and Wu, C.B. (2018) Research of Soil Moisture Content Forecast Model Based on Reference Evapotranspiration in Neighboring Periods. 7th International Conference on Argo-geoinformatics. In: Zhejiang. 1-6.

4. Ji, R.H., Li, X., and Zhang, S.L. (2016) Short-term Prediction of Soil Moisture in Field Based on GM (1, 1) Model Group. Transactions of The Chinese Society of Agricultural Machinery. 401-407.

5. Ma, J.Q. (2013) Real-time irrigation theory and integrated management system for agricultural water resources. China Water\&Power Press. Beijing.

6. Wang, H. (2019) Research on Typical Crops of Economic Irrigation Lower Limit for Drip Irrigation in Greenhouse [D]. Tianjin Agricultural University. 